included group cohesion, within-team communication and team job performance.

Methods Two regional worksites from one large company took part in either the team sport intervention $(n=28)$ or the control group $(n=20)$. The intervention consisted of weekly 1 hour team sport sessions for 12 weeks. Measures of aerobic fitness, physical activity, group cohesion, interaction and performance were measured pre- and post-intervention. Data were analysed using a series of mixed ANOVAs.

Results After 12 weeks, significant improvements were observed in the intervention group in VO2 max $(+4.5$ $\pm 5.8 \mathrm{ml} / \mathrm{min} \mathrm{kg}, \mathrm{p}<0.002)$, interpersonal communication within teams $(+3 \%, \mathrm{p}<0.042)$ and mean weekly physical activity duration $\left(+154.74^{\prime}, \mathrm{p}<0.002\right)$ in the intervention group.

Discussion Participation in team sport might be not only be an effective way to improve aerobic fitness and physical activity behaviour of employees, but may also improve interpersonal communication between colleagues, which may in turn impact organisational well-being. Further workplace team sports studies are required that assess other important indicators of health and social wellbeing.

\section{5b DO HIGH LEVELS OF OCCUPATIONAL SITTING TIME PREDICT SICKNESS ABSENCE, SICKNESS PRESENTEEISM AND LOW WORK ENGAGEMENT OVER TIME?}

F Munir*, I Wilson. Loughborough University, Loughborough UK

\subsection{6/oemed-2018-ICOHabstracts.735}

Introduction Sedentary behaviour, has emerged as a risk factor for premature death and several chronic diseases. About onethird to half of our daily sitting time occurs at work. There is limited research on the link between occupational sitting and important work-related outcomes such as work engagement, presenteeism and sickness absence. An earlier cross-sectional study by Munir, et al. (2015) found that women had higher occupational sitting times than men and that men with high work engagement of vigour and dedication were less likely to have prolonged sitting time. In this study, we examine the effects of occupational sitting time on sickness absence, sickness presenteeism and work engagement, and over an 18 month period.

Methods A cohort of 1005 office workers from the Northern Ireland Civil Service (Stormont) completed a questionnaire in 2012 (T1) and in 2014 (T2). Occupational sitting time were divided into tertiles of low ( $<360$ mins), medium (361-420 mins) and high levels of sitting time (421-600 mins). Logistic regressions and generalised linear regressions were used to analysed data.

Results Participants were predominantly female ( $n=613,61 \%)$. There were no significant findings for occupational sitting times predicting sickness absence. Overall, males who reported moderate levels of sitting times at $\mathrm{T} 1$ were more likely to report engaging in sickness presenteeism at T2. This was not the case for those males reporting high levels of sitting time. Increase sitting time at T1 also contributed to lower levels of work engagement of dedication at $\mathrm{T} 2$ for males. There were no significant findings for females between occupational sitting times and work-related outcomes.
Discussion Our finding findings for levels of sitting time, sickness presenteeism and work engagement warrants further research.

\section{UPDATE ON ASSESSING RISK FOR UPPER LIMB MUSCULOSKELETAL DISORDERS}

David Rempel* ${ }^{*}$. Division of Occupational and Environmental Medicine, University of California at San Francisco, USA

\subsection{6/oemed-2018-ICOHabstracts.736}

The aim of this Special Session is to discuss recent changes to risk assessment tools for upper extremity MSDs.

This session will present recent findings from large prospective epidemiologic studies on carpal tunnel syndrome and other upper extremity disorders. Then other presenters will discuss how those findings have modified common risk assessment models such as the Strain Index, the ACGIH Hand Activity Level, and other methods.

R. Bonfiglioli ${ }^{2}$, B. Evanoff ${ }^{3}$, D. Rempel ${ }^{1}$, J. Kapellusch ${ }^{4}$, B. Weber $^{5}$

${ }^{2}$ Department of Medical and Surgical Sciences, University of Bologna, Italy

${ }^{3}$ Division of General Medical Science, Washington University School of Medicine, Saint Louis, MO, USA

${ }^{4}$ University of Wisconsin - Milwaukee, Milwaukee, USA

${ }^{5}$ Institute for Occupational Safety and Health of the German Social Accident Insurance (IFA), Sankt Augustin, Germany

\section{1a UPDATE ON PHYSICAL FACTORS FROM THE ITALIAN OCTOPUS STUDY}

R Bonfiglioli*. Department of Medical and Surgical Sciences, University of Bologna, Italy

\subsection{6/oemed-2018-ICOHabstracts.737}

Introduction The American Conference of Governmental Industrial Hygienists (ACGIH) proposed a method to assess the hand, wrist and forearm biomechanical overload based on exertions frequency (hand-activity level) and force use (normalised peak force). We applied the ACGIH threshold limit value (TLV) method to a large occupational cohort to assess its ability to predict carpal tunnel syndrome (CTS) onset.

Methods A cohort of industrial and service workers was followed-up between 2000 and 2011. We investigated the incidence of CTS symptoms and CTS confirmed by nerve conduction studies (NCS). We then classified exposure with respect to action limit (AL) and TLV. Cox regression models including age, gender, body mass index, and presence of predisposing pathologies were conducted to estimate hazard ratios (HR) of CTS and population attributable fractions.

Results We analysed data from 3131 workers [females, $\mathrm{n}=2032$ (65\%); mean age at baseline 39.3, standard deviation (SD) 9.4 years]. We observed 431 incident cases of CTS symptoms in 8000 person-years and 126 cases of CTS confirmed by NCS in 8883 person-years. The ACGIH TLV method predicted both CTS symptoms [HR between AL and TLV 2.18, 95\% confidence interval (95\% CI) 1.86 to 2.56 ; above TLV 2.07, 95\% CI: 1.52 to 2.81] and CTS confirmed by NCS (HR between AL and TLV 1.93, 95\% CI: 1.38 to 
2.71; above TLV 1.95, 95\% CI: 1.27 to 3.00). About one third of CTS cases were attributable to exposure levels above the AL.

Conclusions The ACGIH TLV method predicted the risk of CTS, but the dose-response was flat above the AL: a fine-tuning of the proposed thresholds should be considered.

\section{1b RISK FACTORS FOR CARPAL TUNNEL SYNDROME - FINDINGS FROM THE NIOSH UPPER EXTREMITY MUSCULOSKELETAL DISORDER CONSORTIUM}

BA Evanoff* , E Eisen, F Gerr, S Burt, K Hegmann, B Silverstein, A Garg, AM Dale, S Bao, C Harris-Adamson, J Kapellusch, L Merlino, D Rempel. Division of General Medical Science, Washington University School of Medicine, Saint Louis, MO, USA

\subsection{6/oemed-2018-ICOHabstracts.738}

Starting in 2000, six research groups from the USA were supported by NIOSH to perform large, prospective epidemiologic studies examining associations between workplace physical risk factors and upper limb musculoskeletal disorders. A total of 4321 workers at 55 employers/plants across a variety of handintensive industries were followed for up to six years. Individual workplace exposure data included direct observation and video analysis. Health data included self-report, physical examination, and nerve conduction measures; our case definition for carpal tunnel syndrome (CTS) required both typical symptoms and nerve conduction abnormalities. Those performing the physical examinations and the video analyses were blinded to exposure and medical condition, respectively. Pooled analyses of consortium data controlled for personal factors (age, body mass index, gender, co-morbid diseases) and non-overlapping physical exposures (force, posture repetition) to study the association between work exposures and carpal tunnel syndrome. We found no independent effects of wrist posture or total repetition rate on the incidence of CTS. In contrast, strong dose-dependent associations were found between incident CTS and peak hand force (Borg CR10 $\geq 3$ ), forceful repetition rate $(>3$ exertions per minute of $\geq 9 \mathrm{~N}$ pinch force or $45 \mathrm{~N}$ power grip), and the proportion of time spent in forceful exertion $(>11 \%)$. We also found that the ACGIH Threshold Limit Value for Hand Activity (TLV for HAL) predicted CTS, and that that current 'action limit' is too high to adequately protect workers. Varying the formula of the TLV to emphasise force over repetition better predicted incident CTS. Study findings suggest that efforts to reduce workplace exposures should focus on jobs requiring high hand force and repeated or prolonged forceful exertions. Our study also suggests that the TLV for HAL and other less labour intensive assessment methods are valid and usable tools for workplace prevention.

\section{C RECENT CHANGES TO THE ACGIH HAND ACTIVITY LEVEL TLV}

D Rempel*. Division of Occupational and Environmental Medicine, University of California, San Francisco, USA

\subsection{6/oemed-2018-ICOHabstracts.739}

The ACGIH Hand Activity Level (HAL) Threshold Limit Value (TLV) is a risk assessment tool designed to protect workers, who perform repetitive hand exertions for 4 or more hours per day, from distal upper extremity disorders. Recent large, longitudinal studies, provide strong evidence that repetitive forceful hand exertions increase risk for occupational wrist tendinosis and carpal tunnel syndrome. ${ }^{1,2,3}$ In the Harris-Adamson study, ${ }^{3}$ forceful hand exertions (e.g., $>9 \mathrm{~N}$ pinch or $>45 \mathrm{~N}$ power grip force) and the $\%$ time performing forceful hand work increased risk. Based on these and psychophysics studies, ${ }^{4}$ hand exertions should be considered in risk assessment models if they are above $10 \%$ of posture specific strength. In addition, in these large studies, the prior HAL TLV action limit (0.56) was not sufficiently protective ${ }^{5}$ and, therefore, has been revised. The name of this TLV was changed to Hand Activity (HA) TLV. Other changes to the TLV will also be presented.

\section{REFERENCES}

1. Harris C, Eisen E, Goldberg R, et al. Workplace and individual factors in wrist tendinosis among blue-collar workers: The San Francisco study. Scand J Work Environ Health 2011;37(2):86-98.

2. Bonfiglioli $R$, Mattioli $S$, Armstrong $T$, et al. Validation of the ACGIH TLV for hand activity in the OCTOPUS cohort: A two-year longitudinal study of carpal tunnel syndrome. Scand J Work Environ Health 2013;39:155-63.

3. Harris-Adamson C, Eisen EA, Kapellusch J, et al. Biomechanical risk factors for carpal tunnel syndrome: A pooled study of 2474 workers. Occup \& Environ Medicine 2015;72:33-41.

4. Potvin JR. Predicting maximum acceptable efforts for repetitive tasks an equation based on duty cycle. Human Factors 2012;54(2):175-188.

5. Kapellusch JM, Gerr F, Malloy EJ, et al. Exposure-response relationships for the ACGIH TLV for hand activity level: Results from a pooled data study of carpal tunnel syndrome. Scand J Work Environ Health 2014;40:610-20.

\section{1d QUANTIFYING COMPLEX TASKS USING THE REVISED STRAIN INDEX}

JM Kapellusch*, A Garg. University of Wisconsin - Milwaukee, Milwaukee, USA

\subsection{6/oemed-2018-ICOHabstracts.740}

Modern occupational tasks are often complex - consisting of multiple, discrete sub-tasks, each with its own unique combination of force, duration of exertion, posture, and frequency. Quantifying the physical stress from these complex tasks using simple, 'mono-task' assessment tools, such as the 1995 Strain Index (SI), can be difficult and assumes a large degree of ergonomics and MSD knowledge and training. Compared to the 1995 SI, the Revised Strain Index (RSI) minimises complicated measurement decisions and improves upon the 1995 SI by: (1) using percent maximum voluntary contraction (or Borg CR-10 equivalent) for applied hand force, (2) using duration per exertion (in seconds) rather than duty cycle, and (3) distinguishing between flexed and extended wrist postures. Thus the stress from each effort of a task (i.e., each sub task) can be individually quantified by the RSI and compared to other efforts in a cycle, or alternative efforts in the case of task intervention. By incorporating frequency of exertions (i.e., efforts per minute) and duration of task (i.e., hours per day), the RSI summarises stress associated with a simple task in a manner similar to the 1995 SI. For complex tasks, the RSI incorporates the Composite Strain Index (COSI) algorithm which integrates individual sub-tasks into an aggregate COSI score. The algorithm rank-orders subtasks from most to least stressful, thus ensuring that total task-stress is not lower than that of the highest stress sub-task alone. Sub-tasks are then incrementally added to one another by integrating over the frequency of exertion multiplier to produce the final COSI 